\title{
A radiological study of the neural control of oesophageal vestibular function
}

\author{
A. R. CHRISPIN AND G. W. FRIEDLAND \\ From the Hospital for Sick Children, Great Ormond Street, London, W.C.1
}

The oesophageal vestibule lies at the lower end of the oesophagus immediately above the stomach. Pressure recordings in normal adults indicate that after pharyngeal deglutition the vestibule relaxes. They show that this relaxation occurs shortly after deglutition and that it persists until the oesophageal stripping wave reaches the vestibule (Fyke, Code, and Schlegel, 1956). Carré and Astley (1958), studying pressure recordings in normal infants and in infants with hiatal hernia, thought their findings could be interpreted as indicating an intrinsic closing mechanism in the region of the cardia operating independently of the diaphragm'.

Radiological studies in normal infants and children examined in the supine or prone position show that after deglutition a stripping wave passes down the oesophagus, emptying the oesophageal content through the vestibule into the stomach. The vestibule opens as contrast medium passes through it, and it closes as the stripping wave empties the oesophagus. The problem about the oesophagus in the normal infant and child is that the vestibule cannot be studied independently of this stripping wave, and it is difficult to be sure if an intrinsic sphincteric action in the vestibule is present or not. The radiological demonstration of a sphincter depends on showing that no contrast medium flows through a sphincteric zone when the sphincter is closed, but contrast medium must be present proximal to the sphincter for such an observation to be made. An efficient oesophageal stripping wave precludes such studies in normal infants and children.

In this paper an analysis of flow through the oesophageal vestibule in 14 children is presented. These patients have been divided into five groups. A feature common to 10 patients (groups 1 to 3 ) was the absence of an oesophageal stripping wave, and this made it possible to study the vestibule without flow through it being influenced by the propulsive effect of a normal oesophageal strip- $\vec{c}$ ping wave. From the findings it has been deduced $\mathbb{\infty}$ that flow through the vestibule is influenced by $\underset{\mathbb{D}}{+}$ a neural factor.

\section{PATIENTS STUDIED}

GROUP 1 (Fig. 1) This group comprised two patients. with a congenital tracheo-oesophageal fistula without atresia in whom an oesophageal stripping waves was absent. No thoracotomy had been undertaken before the study.

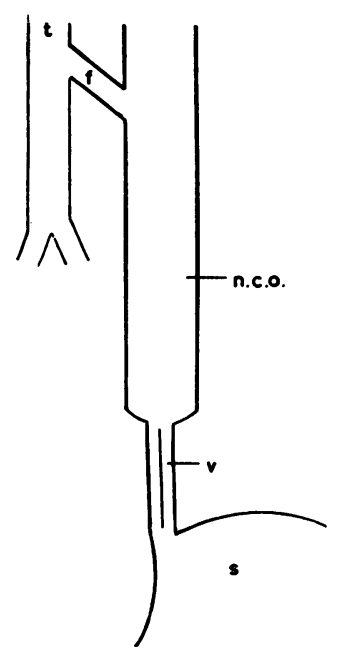

FIG. 1. Group 1. Tracheo-oesophageal fistula; $t$, trachea, $f$, fistula; n.c.o., noncontractile oesophagus; $v$, vestibule; $\mathbb{\mathbb { }}$ $s$, stomach.

GROUP 2 (Fig. 2) In this group were four patients $\frac{\mathbb{D}}{\mathbb{D}}$ who had previously had a technically successful $\underset{\mathbb{D}}{\stackrel{P}{*}}$ repair of an oesophageal atresia with closure of a $\varnothing$ tracheo-oesophageal fistula and in whom the oesophagus was non-contractile above the vestibule. The 


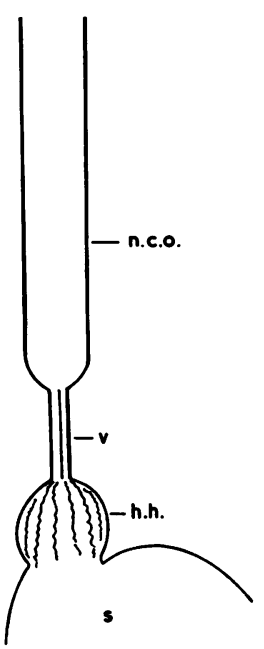

FIG. 2. Group 2. Post-oesophageal atresia repair; n.c.o., noncontractile oesophagus; $v$, vestibule; h.h., hiatal hernia; $s$, stomach.

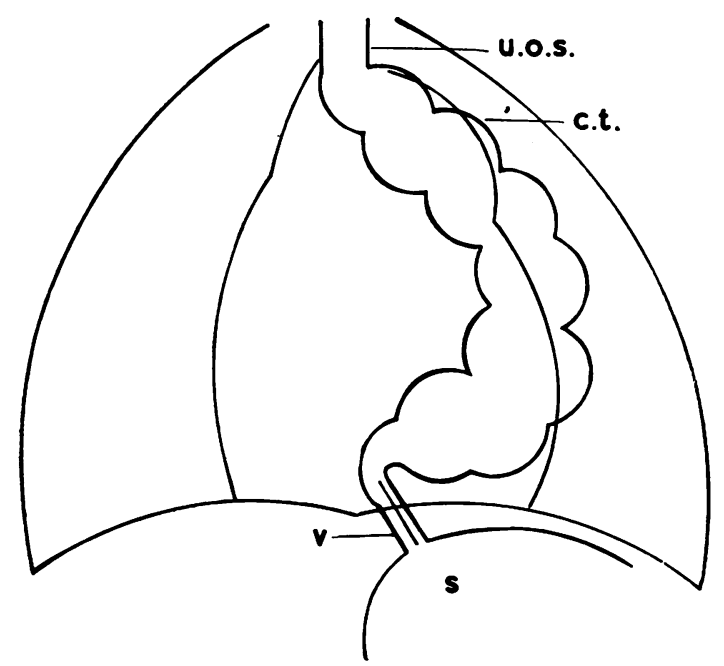

FIG. $3 \mathrm{a}$

FIG. 3. Group 3. Colon transplant for oesophageal atresia; (a) patient supine, P.A.; (b) patient lateral; (c) patient 10 -erect; c.t., colon transplant; v, vestibule; s, stomach; l.h., left hilum; u.o.s., upper oesophageal segment; o, oesophagostomy; e.c., exteriorized colon; f.l., fluid level.

symptoms of these patients and the associated radiological findings have previously been reported (Chrispin, Friedland, and Waterston, 1966).

GROUP 3 (Fig. 3) This group comprised four patients who presented with oesophageal atresia and were treated by colon transplantation. The colon transplant had been interposed above a short lower oesophageal segment. During transplantation no operative pro-

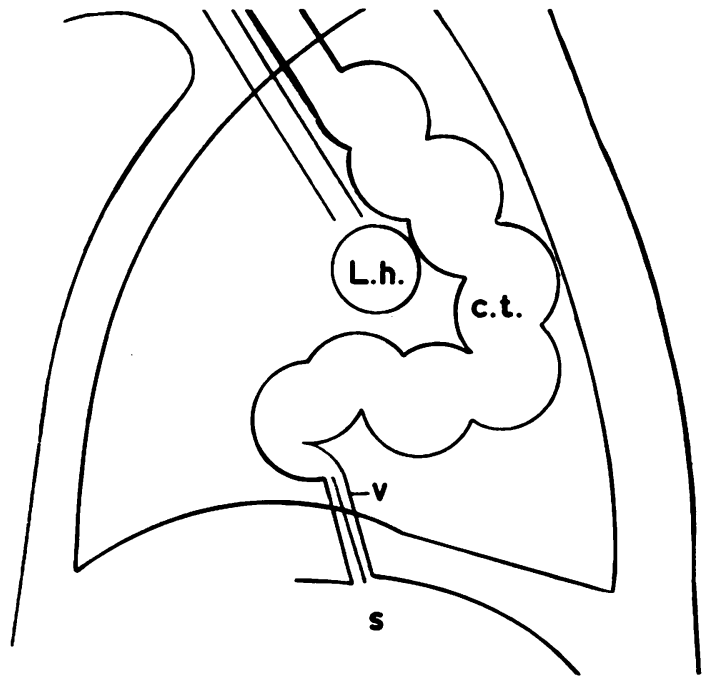

FIG. $3 b$

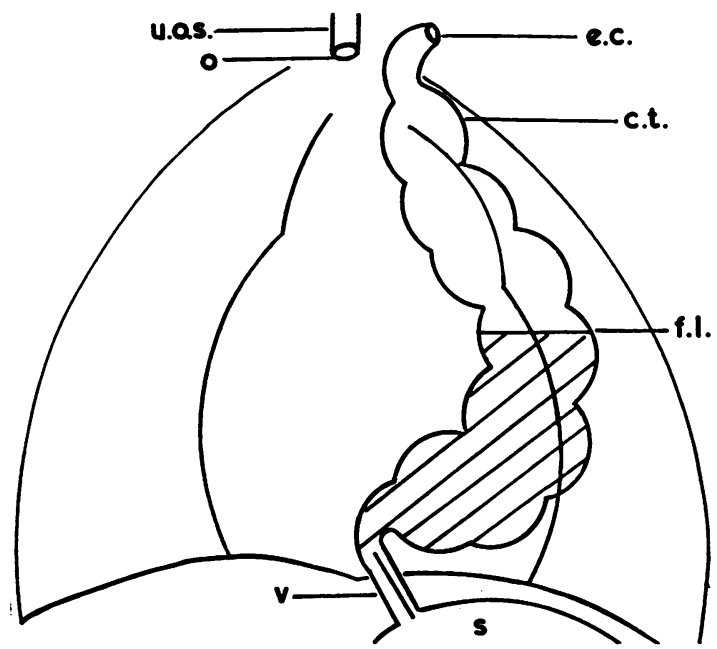

FIG. 3c cedure had been carried out in the immediate vicinity of the vestibule and the oesophageal hiatus.

GROUP 4 (Fig. 4) This group consisted of three patients with a disturbance of the central nervous system associated with vomiting.

GROUP 5 (Fig. 5) These patients were treated surgically for hiatal hernia and stricture by colon transplantation. The proximal anastomosis had been made 


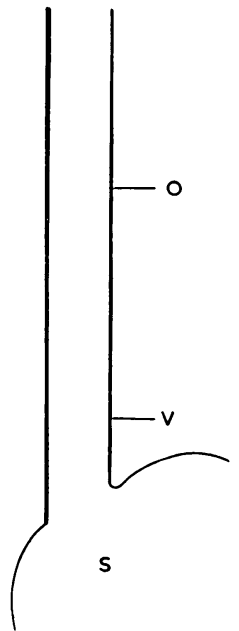

FIG. 4.

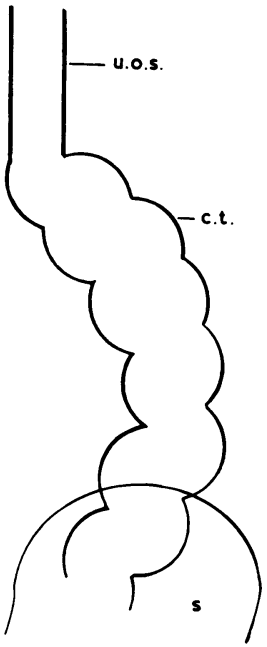

FIG. 5 .
FIG. 4. Group 4. Effect of lesion of central nervous system; o, oesophagus; $v$, vestibule (fails to close); $s$, stomach.

FIG. 5. Group 5. Colon transplant for oesophageal stricture; u.o.s., upper oesophageal segment c.t., colon transplant; s., stomach. between the oesophagus and the colon in the chest. The distal anastomosis lay below the diaphragm and $\frac{\bar{\rho}}{\partial}$ united the colon transplant directly to the stomach. This group has been included because of the absence of any vestibule in the 'gullet', and they are therefore ${ }^{\text {s }}$ useful for comparative purposes. However, only one $\overrightarrow{0}$ patient in this group has been detailed, because the $\vec{\overrightarrow{ }}$ results are being presented separately by our col- $\vec{\omega}$ leagues. Great care was taken to leave the vagi intact in groups 2 and 3.

The cardinal anatomical features of each groupin are given in Figs 1 to 5 . Brief clinical details of the $\overrightarrow{i r}$ patients and the relevant radiological findings are presented in Table I. A very high degret of co-N operation is required from patients in groups 1 to 0 3 during these studies, for crying and sobbing, associ- $\triangle$ ated as they are with repeated acts of deglutition, $\vec{c}$ make a satisfactory assessment impossible.

\section{DISCUSSION}

ANTEGRADE FLOW: RADIOLOGICAL FINDINGS AND INTERPRETATION

Relaxation of vestibule related to deglutition Common to patients in groups 1,2 , and 3 was the finding that the vestibule opened shortly after $\overline{0}$ deglutition and, as the vestibule opened, flow through it into the stomach occurred.

In groups 1 and 2 , and in either the supine or $\overrightarrow{\overrightarrow{0}}$ prone position, flow through the vestibule was

T A B L E I

\begin{tabular}{|c|c|c|c|c|c|c|}
\hline Group & $\begin{array}{c}\text { Patient } \\
\text { No. }\end{array}$ & $\begin{array}{c}\text { Age } \\
\text { (yrs.) }\end{array}$ & $\begin{array}{l}\text { Underlying } \\
\text { Condition }\end{array}$ & $\begin{array}{l}\text { Previous Operative } \\
\text { Procedure }\end{array}$ & $\begin{array}{c}\text { Radiological } \\
\text { Technique }\end{array}$ & Relevant Radiological Findings \\
\hline 1 & $\begin{array}{l}1 \\
2\end{array}$ & $\begin{array}{r}11 \\
6\end{array}$ & $\begin{array}{l}\text { Tracheo-oesophageal } \\
\text { fistula }\end{array}$ & None & $\begin{array}{l}\text { Cine fluoro- } \\
\text { graphy }\end{array}$ & $\begin{array}{l}\text { Whole oesophagus non-contractile; tracheo- } \\
\text { oesophageal fistula; vestibule opened only on } \\
\text { deglutition; when patient } 1 \text { was erect the closed } \\
\text { vestibule supported a column of barium }\end{array}$ \\
\hline 2 & $\begin{array}{l}3 \\
4 \\
5 \\
6\end{array}$ & $\begin{array}{l}6 \\
5 \\
1 \\
4\end{array}$ & $\begin{array}{l}\text { Oesophageal atresia } \\
\text { with fistula }\end{array}$ & $\begin{array}{l}\text { One-stage repair of atre- } \\
\text { sia with closure of } \\
\text { fistula }\end{array}$ & $\begin{array}{l}\text { Fluoroscopy; } \\
\text { also cine } \\
\text { fluorography } \\
\text { in patient } 3\end{array}$ & $\begin{array}{l}\text { Oesophagus non-contractile irom pharynx to } \\
\text { vestibule in patient } 3 \text {, and non-contractile below } \\
\text { anastomosis in patients } 4,5 \text {, and } 6 \text {; vestibule } \\
\text { opened on deglutition; hiatal hernia in patients } \\
4,5 \text {, and } 6 \text {; free reflux in patient } 6\end{array}$ \\
\hline 3 & $\begin{array}{r}7 \\
8 \\
9 \\
10\end{array}$ & $\begin{array}{l}2 \\
6 \\
0 \cdot 8 \\
1 \cdot 8\end{array}$ & $\begin{array}{l}\text { Oseophageal atresia ; } \\
\text { also fistula in } \\
\text { patients } 8 \text { and } 9\end{array}$ & $\begin{array}{l}\text { Anastomosis of colonic } \\
\text { transplant on to short } \\
\text { upper and short lower } \\
\text { oesophageal segment } \\
\text { but only lower anasto- } \\
\text { mosis established in } \\
\text { patient } 10\end{array}$ & $\begin{array}{l}\text { Cine fluoro- } \\
\text { graphy in } \\
\text { patients } 7 \\
8 \text {, and } 10 ; \\
\text { fluoroscopy } \\
\text { in patient } 9\end{array}$ & $\begin{array}{l}\text { No mass movement in colon transplant ; vestibule } \\
\text { opened on deglutition; gravity-assisted empty- } \\
\text { ing when prone or erect; closed vestibule pre- } \\
\text { vented emptying of colon transplant; bi-direc- } \\
\text { tional flow (air upwards, barium downwards) } \\
\text { when vestibule open in patient } 8\end{array}$ \\
\hline 4 & $\begin{array}{l}11 \\
12 \\
13\end{array}$ & $\begin{array}{l}0 \cdot 6 \\
1 \\
2\end{array}$ & $\begin{array}{l}\text { Subdural haemato- } \\
\text { ma; battered baby } \\
\text { syndrome } \\
\text { Cerebral atrophy } \\
\text { Cerebral atrophy } \\
\text { with microcephaly }\end{array}$ & None & Fluoroscopy & $\begin{array}{l}\text { Oesophageal stripping wave inefficient in patients } \\
10,12 ; \text { absent in patient } 11 \text {, vestibule remained } \\
\text { open during investigation with very free reflux } \\
\text { of barium or air }\end{array}$ \\
\hline 5 & 14 & 12 & $\begin{array}{l}\text { Hiatal hernia with } \\
\text { stricture }\end{array}$ & $\begin{array}{l}\text { Colon transplant anasto- } \\
\text { mosed to upper oeso- } \\
\text { phageal segment proxi- } \\
\text { mally and to stomach } \\
\text { distally }\end{array}$ & $\begin{array}{l}\text { Cine fluoro- } \\
\text { graphy }\end{array}$ & $\begin{array}{l}\text { Normal stripping wave in oesophageal segment ; } \\
\text { contractions of colon transplant independent of } \\
\text { oesophagus emptying transplant; some reflux } \\
\text { from stomach; no "empty segment" }\end{array}$ \\
\hline
\end{tabular}


related to deglutition and, when deglutition ceased, the vestibule closed, leaving a residue in the oesophagus above the vestibule. When these patients were erect, gravity-assisted oesophageal emptying occurred, but this was incomplete and at the end of deglutition a gas/fluid level formed in the oesophagus above the closed vestibule. A sliding hiatal hernia was present in three patients in group 2, and from time to time reflux from the stomach into the oesophagus was seen; nonetheless in these patients and in others with no normal oesophageal stripping wave (reported previously-Chrispin et al., 1966) the vestibule lay in the thorax and its opening was related to deglutition. Thus in patients in groups 1 and 2 the closed vestibule, located normally or in the thorax, was able to support a column of fluid when erect and its opening was related to the act of deglutition. Diaphragmatic movement during quiet respiration had no perceptible influence on the closed vestibule.

Effect of a large capacity colon transplant In both groups 1 and 2 it might be thought that the opening of the vestibule was related to shunting of contrast medium associated with forceful pharyngeal deglutition. This seems unlikely in view of the findings in group 3 . In patients 7,8 , and 9 , the colon interposed between the upper and lower oesophageal segments provides a highly distensible viscus whose capacity is much greater than the volume of liquid swallowed at each act of deglutition. The vestibule remained closed except after deglutition. Relaxation took place after deglutition and permitted flow of contrast medium from the transplant through the vestibule into the stomach ; in patient 8 , while the vestibule was relaxed, antegrade flow of contrast medium into the stomach occurred and, at the same time, retrograde flow of gas from the stomach into the transplant was seen, i.e., bi-directional flow during vestibular relaxation. In certain positions it was sometimes possible to see that after swallowing a small quantity of fluid a greater volume (already in the transplant) passed from it into the stomach as the vestibule relaxed.

The findings in patient 10 are important. A colon transplant had been carried out with a distal anastomosis between the transplant and the lower oesophagus. Proximally, the colon had been exteriorized on the left side of the neck. On the right side of the neck an oesophagostomy had been established (Fig. 3c). An open gastrostomy tube and a tube lying in the upper part of the colon transplant permitted studies of the vestibule to be made with both stomach and transplant at what was presumably atmospheric pressure. A little contrast medium was introduced into the colon transplant with the patient in the erect position. When contrast medium was swallowed by mouth it passed through the oesophagus on to the skin of the neck, but shortly after deglutition the vestibule opened, permitting flow from the transplant through the lower oesophageal segment and vestibule and into the stomach. Thus, the relationship between deglutition and opening of the vestibule can be observed even when there is no continuity of lumen between the pharynx and the vestibule.

Interval between deglutition and vestibular opening. It was possible to time the interval between deglutition and vestibular opening in four patients, one having a tracheo-oesophageal fistula and three having a colon transplant (Table II). The

T A B L E II

INTERVAL BETWEEN DEGLUTITION AND VESTIBULAR OPENING

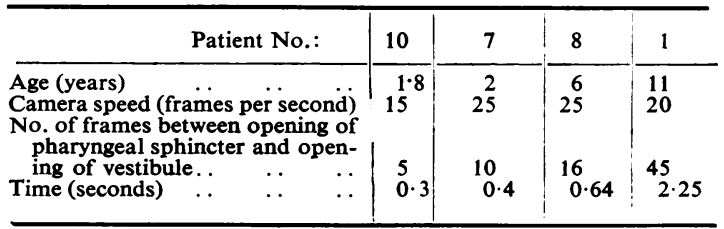

interval lengthened with increasing age and was not related to the capacity of the 'gullet' between the pharynx and the vestibule.

Deglutition initiates relaxation of vestibule by $a$ neural pathway. The opening of the oesophageal vestibule in groups 1,2 , and 3 is related to deglutition. This phenomenon can occur $(a)$ without any previous surgical intervention in the thorax, $(b)$ following a primary anastomosis for an oesophageal atresia-in effect an oesophageal transection, $(c)$ following the interposition of a colon transplant between a short upper oesophageal segment and a short lower oesophageal segment with the transplant disrupting oesophageal continuity and associated with oesophageal transection, and $(d)$ with no continuity of the lumen between the pharynx and the vestibule. The findings are consistent with relaxation of the vestibule being related to deglutition by a neural pathway independent of the oesophagus. Neural pathways involved seem likely to be the IX, X, and XI cranial nerves and may be the sympathetic. 
Effect of a lesion in the central nervous system In patients in group 4 in whom an abnormality of the central nervous system associated with vomiting was present, it was observed that the vestibule remained open during most of the examination of the patient's oesophagus, stomach, and duodenum. Its failure to contract permitted free retrograde flow of contrast medium from the stomach into the oesophagus. Inefficient oesophageal stripping action was present in all patients and antegrade flow down the oesophagus seemed to be largely dependent on pharyngeal deglutition. These findings suggest that the neural pathway has a central component or can be influenced by a central factor.

Lesions involving the human brain stem have been related to abnormalities of oesophageal motility by Meldolesi (1954), Creamer (1962), and Eadie (1963). Such oesophageal abnormalities seem to be associated with lesions of the dorsal motor nucleus of the vagus. In animal studies, selective destruction of vagal motor centres (dorsal motor nucleus and nucleus ambiguus) may result in abnormalities of oesophageal function, but species differences have been noted (Higgs, Kerr, and Ellis, 1965).

RETROGRADE FLOW: RADIOLOGICAL FINDINGS AND INTERPRETATION Ordinarily, except sometimes in early infancy, there is no reflux from the stomach into the oesophagus through a normally placed vestibule. When the vestibule is in the thorax with a sleeve of stomach passing through the hiatus (as in the three patients with a hiatal hernia in group 2), intermittent reflux through the vestibule into the oesophagus may be observed, but the abnormally placed vestibule can still be seen to close. Closure of the vestibule can occur in the patient who has had no previous operation, after a primary anastomosis for oesophageal atresia, and after interposition of a colonic segment above the lower oesophageal segment. Thus the vestibule can close in the absence of continuity of the ozsophagus or of the lumen of the 'gullet' proximally. Its closure can also be observed when the vestibule and the adjacent part of the stomach lie in the thorax. These findings support the opinion that closure of the vestibule occurs as a result of a neural pathway independent of the oesophagus. Failure of closure of the vestibule was seen in the presence of a lesion of the central nervous system.

Patients in group 5, who have no vestibule and have a length of colon below the diaphragm joined directly to the stomach, have all shown reflux from the stomach into the transplant. The $\frac{\overline{\mathrm{O}}}{\mathrm{O}}$ least free retrograde flow was seen in patient $13, \frac{\bar{\sigma}}{\widehat{D}}$ in whom the colon had been anastomosed on to $\varnothing$ the posterior aspect of the fundus of the stomach.ळ It may be that this site of anastomosis provides $a-$ degree of reflux prevention, because the gastric: content lies in the fundus above the transplant $\vec{\omega}$ when the patient is supine. The freest retrogrades flow occurred in a patient in whom the anasto $\overrightarrow{\vec{x}}$ mosis was made on to the apex of the fundus. However, none of the patients in this group hader a so-called 'empty segment'.

\section{CONCLUSIONS}

These studies show the following points:

The vestibule is a functionally definable sphincter permitting flow through it when it opens.

The vestibular sphincter functions in theo absence of a normal oesophageal stripping wave.

Opening and closure of the vestibule occur both in the absence of oesophageal continuity and in the intact oesophagus.

Opening of the vestibule is related to deglutition $\frac{\frac{\pi}{\mathbb{Q}}}{2}$ and is independent of the diaphragm during quiet respiration.

Opening and closure of the vestibule are controlled by a neural pathway linking deglutition to vestibular relaxation.

The interval between deglutition and vestibular opening was timed in four patients, and ix lengthened with increasing age. (In a way this is? to be expected, for the infant has a much shorter oesophagus than the older child. If the time were the same at all ages, difficulties would be encountered after deglutition since intake into the oeso-s phagus could exceed output into the stomach.)

Practical implications include: (a) a logical. approach to the management of patients after aN technically successful repair of oesophagea $\mathrm{h}_{\text {s }}$ atresia (Chrispin et al., 1966) ; (b) a direct colon 은 gastric anastomosis permits reflux from the stomach into the colon, but if an intact vestibule is left between the stomach and the colon (wherfe this is possible) it prevents such reflux; $(c)$ it suggests the importance of the radiological definition of the vestibule before surgery for hiatal hernia in infancy and childhood so that effortsके may be directed to securing a normal relationship $\frac{\text { }}{\mathbb{D}}$ between the vestibule and the stomach, thus per요 mitting efficient functioning of the vestibular sphincter mechanism. 


\section{SUMMARY}

A radiological study of the function of the oesophageal vestibule is presented. Infants and children studied have been divided into five groups; (1) patients with a tracheo-oesophageal fistula; (2) patients after a technically successful repair of oesophageal atresia ; (3) patients treated for oesophageal atresia by interposition of a colon transplant above a short lower oesophageal segment ; (4) patients with a lesion of the central nervous system with vomiting; (5) patients with a severe oesophageal stricture treated by colon transplantation with a direct colon-gastric anastomosis.

Groups 1 to 3 permitted studies of the intact oesophageal vestibule without an associated oesophageal stripping wave. The function of the vestibule as a sphincter is described. Relaxation of the vestibule is related to the act of deglutition. It is deduced that the vestibule is influenced by a neural pathway independent of the oesophagus. In patients with a lesion of the central nervous system associated with vomiting, the vestibule remained open during most of the examination, permitting free reflux from the stomach into the oesophagus. This suggests that the neural pathway has a central component or is influenced by a central factor.
Free reflux from the stomach into the transplant occurred in group 5, showing the value of leaving an intact vestibule between the stomach and colon transplant when this is possible.

We wish to thank our clinical colleagues who have referred patients for examination, and we are particularly indebted to Mr. D. J. Waterston, Professor A. W. Wilkinson, and Mr. G. H. MacNab, who carried out the operations which provide the key to this study. Dr. R. E. Bonham Carter's advice and encouragement in the preparation of this paper have been most helpful.

\section{REFERENCES}

Carré, I. J., and Astley, R. (1958). The gastro-oesophageal junction in infancy. Thorax, 13, 159.

Chrispin, A. R., Friedland, G. W., and Waterston, D. J. (1966). Aspiration pneumonia and dysphagia after technically successful repair of oesophageal atresia. Ibid., 21, 104

Creamer, B. (1962). In Surgical Physiology of the Gastrointestinal Tract: Symposium Royal College of Surgeons of Edinburgh, 1962, Ed. Smith, A. N., p. 50.

Eadie, M. J. (1963). The pathology of certain medullary nuclei in Parkinsonism. Brain, 86, 781.

Fyke, F. E., Code, C. F., and Schlegel, J. F. (1956). The gastrooesophageal sphincter in healthy human beings. Gastroenterologia, 86, 135.

Higgs, B., Kerr, F. W. L., and Ellis, F. H. (1965). The experimental production of esophageal achalasia by electrolytic lesions in the medulla. J. thorac. cardiovasc. Surg., $50,611$.

Meldolesi, G. (1954). L'aspetto radiologico dell'esofago negli encefalitici parkinsoniani; contributo allo studio dell'acalasia atonica dell'esofago. Nunt radiol. (Roma), 20, 567. 